\title{
Brian Activities and Spatial Memory Modulated by CA1 Electrical Stimulation.
}

\section{Elaheh Jafari ${ }^{\star}$ and Hojjatallah Alaei}

Department of Physiology, School of Medicine, Isfahan University of Medical Sciences, Isfahan, Iran

*Corresponding author: Jafari E, Department of Physiology, School of Medicine, Isfahan University of Medical Sciences, Isfahan, Iran, Tel: +98 3137922407; E-mail: jafari.psy.1988@gmail.com

Received date: February 28, 2018; Accepted date: March 13, 2018; Published date: March 20, 2018

\section{Retraction Note:}

The article entitled "Brian Activities and Spatial Memory Modulated by CA1 Electrical Stimulation", has been accepted for publication in the Epilepsy Journal considering the statements provided in the article as personal opinion of the author which was found not having any conflict or biasness towards anything. As the article was a perspective one, information provided by the author was considered as an opinion to be expressed through publication.
Publisher took decision to make the article online solely based on the reviewers suggestion which considered the article not but a personal opinion of the author. However, it is found that the author have some personal concerns and issues, therefore, being retracted from the journal. 